\title{
Dietary Tryptophan and the Risk of Metabolic Syndrome: Total Effect and Mediation Effect of Sleep Duration
}

\section{Weiqi Wang \\ Lin Liu \\ Zhen Tian \\ Tianshu Han \\ Changhao Sun \\ Ying $\mathrm{Li}$}

National Key Discipline, Department of Nutrition and Food Hygiene, School of Public Health, Harbin Medical University, Harbin, People's Republic of China
Correspondence: Changhao Sun; Ying Li National Key Discipline, Department of Nutrition and Food Hygiene, School of Public Health, Harbin Medical University, I57 Baojian Road, Harbin, I5008I, People's Republic of China Tel +86-45 I-8750-280 I

Email changhaosun2002@163.com; liying_helen@163.com
Purpose: Tryptophan affects energy homeostasis, glucose metabolism, blood pressure, and sleep. However, studies investigating the association between tryptophan and metabolic syndrome (MetSyn) are rare. We aimed to investigate the associations of dietary tryptophan with MetSyn incidence and potential mediation via sleep duration.

Methods: Data of 7890 participants were obtained from the China Health and Nutrition Survey (1997-2011) (male: 49.9\%; mean age=43.43 years;median follow-up=129.76 months; MetSyn incidence: $16.3 \%$ ). A combination of individual 24-hour recall and household survey was used to assess dietary intake. In total, 6720 and 4474 participants who reported sleep duration and had blood samples taken, respectively, were incorporated into subgroup analyses. MetSyn was defined according to National Cholesterol Education Program Adult Treatment Panel (NCEP ATP) III criteria (2004), and tryptophan consumption and sleep duration were assessed by self-report in each survey. Multivariate Cox regression models were used to assess the associations between tertiles of tryptophan intake and MetSyn. Generalized linear regression models were used to evaluate the effect of tryptophan on sleep duration and plasma biomarkers.

Results: Dietary tryptophan showed a protective effect on the risk of MetSyn. The hazard ratio $(95 \% \mathrm{CI})$ of MetSyn was $0.77(0.65-0.90)$ for individuals with a high tertile of tryptophan. Sleep duration was significantly higher, and $\mathrm{HbA}_{1 \mathrm{c}}$, total cholesterol (TC), lowdensity lipoprotein cholesterol (LDL-C), and apolipoprotein B (APO-B) were lower in the high tertile of tryptophan compared to the low tertile $(P<0.05)$. In addition, mediation effects on the association between tryptophan intake and MetSyn risk were observed for sleep duration (estimated mediation percentage: $26.5 \%$ ).

Conclusion: Our study demonstrated a negative association between dietary tryptophan and MetSyn incidence, and the mediation effect of sleep duration on this association, after adjusting for numerous confounders such as nutrients and food patterns. These findings may have important public health implications for the improvement of cardiometabolic health.

Keywords: dietary tryptophan, metabolic syndrome, sleep duration, mediation

\section{Introduction}

The metabolic syndrome (MetSyn) is characterized by a multifactorial cardiometabolic disorder, including abdominal obesity, elevated blood pressure, high blood glucose, increased triglycerides, and decreased high-density lipoprotein cholesterol (HDL-C). ${ }^{1,2}$ These cardiometabolic risk factors are associated with increased risk for cardiovascular disease, type 2 diabetes, cancer, and all-cause mortality, independently of other risk factors, and have dramatically increased worldwide. ${ }^{3-5}$ 
Given the enormous health and economic burden imposed by diseases associated with MetSyn, finding effective strategies to prevent or treat MetSyn is a public health issue with high priority. ${ }^{6}$

Tryptophan, an essential component of the diet, serves as the sole precursor of serotonin and plays a vital role in the brain's serotonergic system. ${ }^{7}$ Rapidly accumulating evidence suggests the involvement of the brain's serotonergic system in MetSyn, according to its influences on many behaviors (eg eating, sleep, and stress responses), as well as autonomic functions (eg thermogenesis, cardiovascular control, circadian rhythms, and pancreatic function). ${ }^{8-10}$ Moreover, previous research has linked dietary tryptophan with some component features of MetSyn, such as blood pressure and triglycerides. ${ }^{11,12}$ In strokeprone spontaneously hypertensive rats, a single oral dose of $100 \mathrm{mg} \mathrm{L}$-tryptophan $/ \mathrm{kg}$ body weight could improve blood pressure. ${ }^{11}$ Furthermore, administration of $2.5 \mathrm{~g} / \mathrm{kg}$ diet tryptophan for 7 days in normal rats significantly decreased serum triglyceride levels. ${ }^{12}$ However, no studies have focused on the effect of tryptophan intake on MetSyn incidence. Therefore, a large-scale study is needed to better explore the associations, if any, between tryptophan consumption and MetSyn risk, as well as the potential factors mediating the development of MetSyn.

Sleep loss, be it behavioral or related to sleep disorders, is an increasingly common condition in modern society. ${ }^{13}$ The pooled prevalence of sleeplessness in the general population is $10-48 \%$, and in China it is approximately $15.0 \%$, which is worthy of attention. ${ }^{14,15}$ Both cross-sectional and prospective epidemiological studies have linked short sleep duration with obesity, hypertension, and impaired glucose metabolism. ${ }^{13,16}$ Increased body weight and glucose disorder have pernicious and profound consequences on cardiometabolic risk, including dyslipidemia, enhanced inflammation, and endothelial dysfunction. ${ }^{17}$ Given that inadequate sleep significantly changes the main components of MetSyn, it is not surprising that a meta-analysis consisting of 12 studies showed that short sleep duration was significantly associated with increased risk of MetSyn. ${ }^{18}$ Several population studies, originating from different geographical locations and involving diverse subject populations, have illustrated that tryptophan consumption exerts an impact on sleep time by influencing the hypnotic neurotransmitters serotonin and melatonin, indicating that doses as low as $1 \mathrm{~g}$ L-tryptophan significantly reduce sleep latency and increase subjective ratings of sleepiness in subjects with insomnia. ${ }^{19,20}$ However, the role played by sleep duration in linking dietary tryptophan and
MetSyn has not yet been demonstrated in a large-scale cohort study.

Numerous studies have reported that dietary tryptophan is related to sleep and that sleep can exert effects on MetSyn risk. Furthermore, advances in mediation analysis methods have expanded the modeling possibilities for evaluating potential mechanistic intermediates using longitudinal epidemiological data. ${ }^{21}$ Therefore, we aimed to estimate the association between dietary tryptophan and the incidence of MetSyn using longitudinal data from the China Health and Nutrition Survey (CHNS), as well as to determine whether sleep duration partially mediates this association, through mediation analysis. It is hoped that our research will contribute to a better understanding of the effect of dietary tryptophan intake on MetSyn incidence and the potential mediation mechanisms, and provide a new dietary therapy in clinical practice to prevent and manage MetSyn more effectively.

\section{Materials and Methods Ethical Approval}

This study was conducted in accordance with the Declaration of Helsinki. It was organized by the Carolina Population Center at the University of North Carolina at Chapel Hill and the National Institute for Nutrition and Health (former National Institute of Nutrition and Food Safety) at the Chinese Center for Disease Control and Prevention (CCDC). On this basis, the survey protocols, instruments, and the process for obtaining informed consent were approved by these two institutes. All participants provided written informed consent prior to the surveys.

\section{Study Population}

Details of the CHNS are described elsewhere. ${ }^{22}$ In brief, the CHNS is a longitudinal household survey designed to investigate health and nutritional status in Chinese populations, and to reflect the effects of social, economic, and demographic transformations in China on health and nutritional status. The study used a multistage random-cluster sampling process to select participants from nine provinces varying according to geography, economic development, and health indicators, and involved eight surveys from 1991 to $2011 .^{23}$ Each survey maintained the desired range of sociological, economic, and demographic variables. In 1997, 14,441 participants completed the survey in the CHNS. We analyzed CHNS data from 1997 to 2011, and the following individuals were excluded: 4059 
participants who were less than 18 years old at baseline; 1888 participants who were lost to follow-up and participated in only one survey; 540 participants who had implausible energy intake ( $<500$ or $>5000 \mathrm{kcal} /$ day) 46 participants who were pregnant; and 18 participants who diagnosed with MetSyn at baseline. Finally, 7890 adults were selected as the study subjects, and the newly diagnosed MetSyn cases during follow-up were included in the case group.

\section{Questionnaire Survey}

Detailed in-person interviews were administered by trained personnel using a structured questionnaire to collect information on demographic characteristics, dietary habits, lifestyle, physical condition, and anthropometric characteristics. Dietary assessment was based on a combination of three consecutive 24-hour recalls at the individual level, and a food inventory taken at the household level over the same 3-day period, which has been shown to improve the accuracy of recall. ${ }^{24}$ Individuals were required to report all foods and beverages consumed both at home and away from home on a 24-hour recall basis, ${ }^{25}$ which has been previously validated for energy intake using double-labeled water. $^{26}$ At the household level, interviewers estimated the amount of each dish prepared from the household inventory and the proportion of each dish consumed by each individual, to further estimate the individual salt and oil intake. Detailed dietary data collection is described elsewhere. ${ }^{27}$ The Chinese Food Composition Table (1991) was utilized to calculate individual daily intake of select nutrients for each food item in the dietary data for 1997 and 2000. A combined version of the Food Composition Table (2002 and 2004) was used for the surveys from 2004 to 2011. The tryptophan content of some food items was not measured and was replaced by the mean tryptophan of their food group or similar foods. Sleep duration as defined by the answer to the question "How many hours each day do you usually sleep, including during both daytime and nighttime?" Current smoking was defined as a positive answer to the question "Do you still smoke cigarettes or a pipe?", and alcohol consumption was measured using the question, "During the past year, what was your consumption (frequency and quantity) of beer, liquor and wine?" Physical activity level (PAL) was defined as the combination of occupational activity and home activity, as previously reported. ${ }^{28}$ The total metabolic equivalents (METs) of physical activity were calculated as MET-h per week.

\section{Anthropometric Measurements and Biochemical Analyses}

In each survey, height was measured without shoes to the nearest $0.2 \mathrm{~cm}$ using a portable SECA stadiometer (SECA, Hamburg, Germany). Weight was measured without shoes and in light clothing to the nearest $0.1 \mathrm{~kg}$ using a calibrated beam scale. Body mass index (BMI) was calculated as weight $(\mathrm{kg})$ divided by the square of the height in meters $\left(\mathrm{m}^{2}\right)$. Waist-to-hip ratio (WHR) was calculated as waist $(\mathrm{cm})$ divided by hip $(\mathrm{cm})$ circumference. A $12 \mathrm{~mL}$ blood sample was collected after overnight fasting in the survey in 2009. Serum glucose was tested using the GOD-PAP method (Hitachi 7600; Randox, UK). Whole blood $\mathrm{HbA}_{1 \mathrm{c}}$ HPLC analysis (PDQ A1c; Primus, USA) generated continuous outcomes for $\mathrm{HbA}_{1 \mathrm{c}}$. Triacylglycerol (TG) was measured using the glycerolphosphate oxidase (GPO-PAP) method (Kyowa, Tokyo, Japan). Total cholesterol (TC) was measured using the CHOD-PAP method (Hitachi 7600; Kyowa, Japan). LDLcholesterol (LDL-C) was measured using enzymatic methods (Hitachi 7600; Kyowa Japan). Apolipoprotein B (APO-B) was measured using the immunoturbidimetric method (Hitachi 7600; Randox, UK).

\section{Case Ascertainment}

MetSyn was defined according to the revised National Cholesterol Education Program Adult Treatment Panel (NCEP ATP) III criteria (2004), with modification of the waist circumference cut-off to be more appropriate for an Asian population. ${ }^{29,30}$ MetSyn was diagnosed when three or more of the following criteria were met in a participant: 1) blood pressure $\geq 130 / 85 \mathrm{mmHg}$ or taking antihypertensive drugs; 2) waist circumference $\geq 90 \mathrm{~cm}$ in men and $\geq 80 \mathrm{~cm}$ in women; 3) triglyceride level $\geq 1.69 \mathrm{mmol} / \mathrm{L}$ (150 mg/dL); 4) HDL-C level $<1.03 \mathrm{mmol} / \mathrm{L}$ (40 mg/dL) in men and $<1.29 \mathrm{mmol} / \mathrm{L}(50 \mathrm{mg} / \mathrm{dL})$ in women; and 5 ) fasting plasma glucose (FPG) level $\geq 5.6 \mathrm{mmol} / \mathrm{L}(100 \mathrm{mg} /$ $\mathrm{dL})$ or taking hypoglycemic medications.

\section{Statistical Analysis}

Tryptophan intake was highly correlated with total protein intake $(r=0.79, P<0.001)$ in partial correlation analysis. Therefore, nutrient densities were used to reduce the likelihood of multicollinearity, by which the tryptophan intake was characterized as a proportion of total protein intake. ${ }^{31}$ Then, tryptophan intake was modeled as a cumulative average of intake through the questionnaire preceding the 
diagnosis and was stratified into tertiles, which provide the most stable estimate of adult diet in studies involving repeated measurements. ${ }^{32}$ For covariates that were missing for fewer than $5 \%$ of participants, the missing values were replaced by the median values. For each disease-specific analysis, prevalent cases of the corresponding disease were excluded.

Cox multivariate regression models were used to estimate associations between tryptophan intake and the incidence of MetSyn. Time at entry was the age at the beginning of follow-up, and exit time was the age when participants were diagnosed as cases, lost to follow-up, or censored at the end of the follow-up period, whichever came first. Models were adjusted for sex, age, BMI, WHR, living site, smoking status, drinking status, physical activity, urban index, cardiovascular disease, Alternate Healthy Eating Index (AHEI), saturated fatty acid (SFA), fiber, sugar, fruit, vegetable, and energy intake. Interactions were tested between tryptophan intake and the following variables on MetSyn incidence: sex, age, WHR, overweight/obesity, abdominal obesity, and living site. $P$ for interaction was obtained by introducing a cross-product interaction term in the multivariate model. For statistically significant interactions, corresponding stratification analyses were performed. In sensitivity analysis, considering potential confounding caused by the overlap between dietary measurements (1997-2011) and blood collections (2009), we assessed the association between dietary tryptophan and MetSyn incidence after excluding the 2011 survey.

To investigate the relationship between tryptophan intake and sleep time and some blood biomarkers, subgroup analysis was performed in 6720 participants who reported their sleep time and 4474 who had a blood sample taken in 2009. Multivariable generalized linear models and linear regression models were performed to test differences in sleep time and biomarkers across tryptophan intake with adjustment for the above covariates. After a significant association between tryptophan intake and the risk of MetSyn was established, and differences in sleep time and plasma biochemical factors were confirmed, a mediation analysis model was constructed to examine whether the association between tryptophan intake and risk of MetSyn was mediated by sleep time after adjusting for above covariates. Mediation analysis was performed using $\mathrm{R}$ package lavaan. ${ }^{33}$

Generalized linear models were performed to examine the differences in baseline characteristics of continuous variables across tertiles of tryptophan consumption. The $\chi^{2}$ test was used to measure differences in baseline characteristics of classified variables. Statistical analyses were performed using R 3.4 .3 (www.r-project.org/) and SPSS 23.0 (Beijing Stats Data Mining Co. Ltd, Beijing, China). A two-sided $P$-value $<0.05$ was considered statistically significant.

\section{Results}

\section{Description of the Study Population}

A total of 1290 MetSyn cases were identified in the CHNS cohort from 1997 to 2011. Characteristics of the overall study population by survey year are presented in Supplementary Table 1. Waist, WHR, and total dietary fat intake showed increasing trends across the survey years, whereas tryptophan and total energy intake showed decreasing trends. For the components of MetSyn, the prevalence of blood pressure-related components $(65.3 \%)$ was higher than the other components (waist related: 55.1\%; TG related: $17.5 \%$; HDL-C related: $12.9 \%$; FPG related: $18.0 \%$ ). The general demographic characteristics according to tertiles of tryptophan intake are summarized in Table 1. Compared to the first tertile, participants in the last tertile had longer sleep duration; lower BMI, waist circumference, WHR, SBP, DBP, and energy intake; and were less likely to be current drinkers and to have MetSyn. Furthermore, at baseline, people with diabetes, obesity, overweight/obesity (BMI $\geq 24 \mathrm{~kg} / \mathrm{m}^{2}$ ), and hypertension were less likely to appear in the last tertile.

\section{Associations Between Tryptophan Intake and the Risk of MetSyn}

The hazard ratios (HRs) and 95\% confidence intervals (CIs) for MetSyn according to tertiles of tryptophan are shown in Table 2. In the crude Cox model, a high intake of tryptophan was inversely associated with the risk of MetSyn ( $P$ for trend $<0.001$ ). After controlling for sex, age, BMI, WHR, living site, smoking status, drinking status, physical activity, urban index, cardiovascular disease, AHEI, SFA, fiber, sugar, fruit, vegetable, and energy intake, compared to the lowest intake, those with the highest intake of tryptophan had a significantly, 23\% [HR (95\% CI) 0.77 (0.65-0.90)], lower risk of MetSyn. The trend toward a greater reduction in the risk of MetSyn with increasing tryptophan consumption was also of significance $(P$ for trend $=0.003)$. When dietary tryptophan was 
Table I Baseline Characteristics of Study Variables by Tertiles of Cumulative Tryptophan Intake in the CHNS, 1997-20I I

\begin{tabular}{|c|c|c|c|c|c|}
\hline \multirow[t]{2}{*}{ Baseline Variable } & \multirow{2}{*}{$\begin{array}{l}\text { Overall } \\
\text { Participants }\end{array}$} & \multicolumn{3}{|c|}{ Tertiles of Dietary Tryptophan Intake } & \multirow[t]{2}{*}{ P-Value } \\
\hline & & TI $(n=2624)$ & T2 $(n=2636)$ & T3 $(n=2630)$ & \\
\hline Baseline tryptophan consumption (mg/g protein) & $13.45(1.04)$ & $12.29(0.52)$ & $13.49(0.25)$ & $14.56(0.56)$ & $<0.001$ \\
\hline MetSyn cases, n (\%) & $1290(16.3)$ & $527(20.1)$ & $457(17.3)$ & $306(11.6)$ & $<0.001$ \\
\hline Age (years) & $43.43(14.85)$ & $43.82(14.60)$ & $43.15(14.77)$ & $43.32(15.18)$ & 0.23 \\
\hline Female, n (\%) & $3951(50.1)$ & $1290(49.2)$ & $1334(50.6)$ & $1327(50.5)$ & 0.52 \\
\hline BMI $\left(\mathrm{kg} / \mathrm{m}^{2}\right)$ & $22.27(2.89)$ & $22.81(2.96)$ & $22.33(2.93)$ & $21.66(2.66)$ & $<0.001$ \\
\hline Waist (cm) & $77.38(8.61)$ & $79.04(8.86)$ & $77.59(8.60)$ & $75.51(8.00)$ & $<0.001$ \\
\hline WHR & $0.85(0.063)$ & $0.85(0.065)$ & $0.85(0.06 \mathrm{I})$ & $0.84(0.06 \mathrm{I})$ & $<0.001$ \\
\hline PAL (MET-h/week) & $308.96(182.85)$ & $306.28(186.00)$ & $305.71(183.87)$ & $314.90(178.52)$ & 0.12 \\
\hline Energy intake (kcal/day) & $2232.52(663.44)$ & $2406.32(728.30)$ & $2279.18(631.69)$ & $23 \mid 2.37(6 \mid 8.83)$ & $<0.001$ \\
\hline Protein intake (g/day) & $68.99(22.37)$ & $75.86(24.45)$ & $67.99(21.01)$ & $63.14(19.51)$ & $<0.001$ \\
\hline Fat intake (g/day) & $65.54(35.89)$ & $64.87(37.47)$ & $71.46(36.82)$ & $60.26(32.28)$ & $<0.001$ \\
\hline Carbohydrate intake (g/day) & $363.64(126.09)$ & $376.97(142.76)$ & $337.91(113.61)$ & $376.13(115.92)$ & $<0.001$ \\
\hline $\mathrm{SBP}(\mathrm{mmHg})$ & $118.63(17.72)$ & $120.85(17.8)$ & $118.30(17.87)$ & $116.79(17.25)$ & $<0.001$ \\
\hline $\mathrm{DBP}(\mathrm{mmHg})$ & $77.03(10.85)$ & $78.25(10.74)$ & $77.02(11.26)$ & $75.84(10.39)$ & $<0.001$ \\
\hline Living in city, n (\%) & $2273(28.8)$ & $759(28.9)$ & $936(35.5)$ & $578(22.0)$ & $<0.001$ \\
\hline Urban index & $51.92(2.77)$ & $51.95(2.96)$ & $52.03(2.73)$ & $51.79(2.62)$ & 0.005 \\
\hline High school education, n (\%) & $1369(17.4)$ & $454(17.3)$ & $568(21.5)$ & $347(13.2)$ & $<0.00$ I \\
\hline Smoking, n (\%) & $2627(33.3)$ & $885(33.7)$ & $889(33.7)$ & $853(32.4)$ & 0.52 \\
\hline Drinking, n (\%) & $2903(36.8)$ & $1006(38.3)$ & 994 (37.7) & $903(34.3)$ & 0.005 \\
\hline Sleep time $(\mathrm{h})$ & $8.13(1.16)$ & $8.08(1.13)$ & $8.10(1.18)$ & $8.21(1.16)$ & $<0.001$ \\
\hline Prevalent diabetes, n (\%) & I0I (I.3) & $23(0.9)$ & $32(1.0)$ & $46(1.7)$ & 0.018 \\
\hline Prevalent obesity, n (\%) & $344(4.4)$ & $162(6.2)$ & $114(4.4)$ & $69(2.2)$ & $<0.001$ \\
\hline Prevalent overweight/obesity, n (\%) & $1273(16.1)$ & $534(20.4)$ & $467(17.7)$ & $272(10.3)$ & $<0.001$ \\
\hline Prevalent hypertension, n (\%) & $1409(17.9)$ & $548(20.9)$ & $473(17.9)$ & $388(14.8)$ & $<0.001$ \\
\hline
\end{tabular}

Notes: Continuous data are expressed as mean (SD) or number (\%); generalized linear models and $\chi^{2}$ test were used to probe for differences in continuous variables and dichotomous variables.

Abbreviations: MetSyn, metabolic syndrome; BMI, body mass index; WHR, waist-to-hip ratio; PAL, physical activity level; SBP, systolic blood pressure; DBP, diastolic blood pressure.

shown in a continuous form, the hazard ratio of MetSyn per $1 \mathrm{mg} / \mathrm{g}$ protein increment of tryptophan was $0.91(95 \%$ CI: $0.86,0.97)$. In the interaction analysis, except for overweight/obesity ( $P$ for interaction $=0.002$ ), no significant modification was found with regard to age $(P$ for interaction=0.23), sex $(P$ for interaction=0.66), WHR ( $P$ for interaction $=0.63)$, abdominal obesity $(P$ for interaction $=0.09)$, or living site $(P$ for interaction $=0.51)$. Analysis stratified according to overweight/obesity $(<24$ or $\geq 24)$ showed that tryptophan greatly decreased MetSyn risk in those without overweight/obesity [HR $\left(\begin{array}{llll}95 \% & \mathrm{CI} & 0.62\end{array}\right.$ $(0.50-0.77)]$, but not in overweight/obese participants (Supplementary Table 2). Furthermore, in sensitivity analysis excluding the 2011 survey, we observed similar associations between tryptophan intake and the risk of MetSyn (Supplementary Table 3). 
Table 2 Associations Between the Cumulative Consumption of Dietary Tryptophan and the Risk of MetSyn, from Cox Proportional Hazards Models, CHNS, 1997-20II

\begin{tabular}{|c|c|c|c|c|c|c|}
\hline & \multicolumn{3}{|c|}{ Tertiles of Cumulative Dietary Tryptophan Intake } & \multirow[t]{2}{*}{$P$ for Trend } & \multirow[t]{2}{*}{ Per I mg/g Protein Increment } & \multirow[t]{2}{*}{ P-Value } \\
\hline & TI & T2 & T3 & & & \\
\hline Cases, n (\%) & $527(20.1)$ & $457(17.3)$ & $306(11.6)$ & & $1290(16.3)$ & \\
\hline \multicolumn{7}{|l|}{ Model I } \\
\hline $\mathrm{HR}(95 \% \mathrm{Cl})$ & & $0.88(0.77-0.99)$ & $0.63(0.55-0.72)$ & $<0.001$ & $0.84(0.80-0.89)$ & $<0.001$ \\
\hline \multicolumn{7}{|l|}{ Model 2} \\
\hline HR $(95 \% \mathrm{Cl})$ & 1 & $0.88(0.78-1.00)$ & $0.63(0.54-0.72)$ & $<0.001$ & $0.84(0.80-0.89)$ & $<0.001$ \\
\hline \multicolumn{7}{|l|}{ Model 3} \\
\hline $\mathrm{HR}(95 \% \mathrm{Cl})$ & 1 & $0.98(0.86-1.11)$ & $0.78(0.68-0.90)$ & 0.001 & $0.92(0.87-0.97)$ & 0.004 \\
\hline \multicolumn{7}{|l|}{ Model 4} \\
\hline $\mathrm{HR}(95 \% \mathrm{Cl})$ & 1 & $0.98(0.86-1.1 I)$ & $0.78(0.68-0.90)$ & 0.001 & $0.92(0.87-0.97)$ & 0.003 \\
\hline \multicolumn{7}{|l|}{ Model 5} \\
\hline HR ( $95 \% \mathrm{Cl})$ & 1 & $0.99(0.87-1.12)$ & $0.79(0.69-0.91)$ & 0.002 & $0.92(0.87-0.98)$ & 0.004 \\
\hline \multicolumn{7}{|l|}{ Model 6} \\
\hline $\mathrm{HR}(95 \% \mathrm{Cl})$ & 1 & $0.99(0.87-1.15)$ & $0.77(0.65-0.90)$ & 0.003 & $0.91(0.86-0.97)$ & 0.005 \\
\hline
\end{tabular}

Notes: Model I was the crude Cox proportional hazards models without adjusting for any covariate; Model 2 was further adjusted for age and sex; Model 3 was further adjusted for WHR and BMI; Model 4 was further adjusted for smoking and drinking status; Model 5 was further adjusted for living site, physical activity, urban index, cardiovascular disease, and energy intake; Model 6 was adjusted for all variables in model 5, with further adjustment for AHEI, SFA, fiber, sugar, fruit, and vegetable intake. Abbreviations: MetSyn, metabolic syndrome; WHR, waist-to-hip ratio; BMI, body mass index; AHEI, Alternate Healthy Eating Index; SFA, saturated fatty acid.

\section{Associations Between Tryptophan Consumption and Sleep Time and Plasma Biomarkers}

Differences in sleep duration and plasma biomarkers across tertiles of tryptophan consumption are shown in Table 3. Compared to the first tertile, participants in the last tertile seemed to have longer sleep time ( $P$ for trend $=0.033)$. The increment of sleep time per grade of dietary tryptophan was $0.042(0.020)$. When tryptophan was changed into a continuous form, a positive correlation was still observed between tryptophan consumption and sleep duration according to the multiple linear regression model $(P=0.001)$.

As for biochemical factors in plasma, changes in FPG, $\mathrm{HbA}_{1 \mathrm{c}}, \mathrm{TC}, \mathrm{LDL}-\mathrm{C}$, and APO-B per tertile of tryptophan were $-0.091 \quad(0.030),-0.148 \quad(0.020)$, -0.072 (0.021), $-0.081(0.020)$, and $-0.021(0.005)$ respectively (all $P$ for trend $<0.05$ ). Similar tendencies of these biomarkers were found when tryptophan intake was converted into a continuous variable (Table 3).

\section{Mediation Analysis}

Figure 1 shows the mediation effects of sleep time on the association between tryptophan consumption and the risk of MetSyn. The total effect of tryptophan consumption was estimated at $-0.166(P<0.05)$. The indirect effect for sleep time was calculated by $\beta 1$ and $\beta 2$ ( $\beta$ ind $=-0.044$, $P<0.05)$. The percentage of the total effect mediated by sleep time was estimated at $26.5 \%$.

In sensitivity analyses, after excluding the extremes of $1 \%$ outliers for sleep duration or more than 10 hours of sleep time in sensitivity analyses, we observed a similar but weaker mediation effect of sleep duration on the association between dietary tryptophan and MetSyn incidence (Supplementary Figures 1 and 2). After excluding the extremes of $1 \%$ outliers, the percentage of the total effect mediated by sleep time was estimated at $22.9 \%$. After excluding participants with more than 10 hours of sleep time, the percentage changed to $24.7 \%$.

\section{Discussion}

In this large prospective study, we found that participants in the highest tertile of cumulative intake of dietary 
Table 3 Differences in Sleep Time and Blood Biochemical Factors Across Cumulative Dietary Tryptophan Intake

\begin{tabular}{|l|l|l|l|l|l|l|l|}
\hline & \multicolumn{3}{|l}{ Dietary Tryptophan Intake in Tertiles } & \multicolumn{2}{l}{$\begin{array}{l}\text { Dietary Tryptophan Intake in } \\
\text { Continuous Form }\end{array}$} \\
\cline { 2 - 8 } & TI & T2 & T3 & Effect Size & P for Trend & Effect Size & P-Value \\
\hline Sleep time (h) & $8.10(0.027)$ & $8.12(0.025)$ & $8.19(0.027)$ & $0.042(0.020)$ & 0.033 & $0.055(0.016)$ & 0.001 \\
\hline FPG (mmol/L) & $5.58(0.040)$ & $5.38(0.038)$ & $5.39(0.042)$ & $-0.091(0.030)$ & 0.003 & $-0.097(0.021)$ & $<0.001$ \\
\hline HbA Ic (\%) & $5.86(0.026)$ & $5.56(0.025)$ & $5.56(0.027)$ & $-0.148(0.020)$ & $<0.001$ & $-0.152(0.016)$ & $<0.001$ \\
\hline TC (mmol/L) & $4.98(0.027)$ & $4.92(0.026)$ & $4.84(0.028)$ & $-0.072(0.021)$ & $<0.001$ & $-0.063(0.017)$ & $<0.001$ \\
\hline LDL-C (mmol/L) & $3.11(0.026)$ & $2.99(0.025)$ & $2.95(0.028)$ & $-0.081(0.020)$ & $<0.001$ & $-0.071(0.016)$ & $<0.001$ \\
\hline APO-B (g/L) & $0.93(0.007)$ & $0.89(0.007)$ & $0.88(0.007)$ & $-0.021(0.005)$ & $<0.001$ & $-0.018(0.004)$ & $<0.001$ \\
\hline
\end{tabular}

Notes: Multivariable generalized linear models were used to probe for differences across tertiles of tryptophan consumption with adjustment for sex, age, BMI, WHR, smoking status, drinking status, living site, physical activity, urban index, cardiovascular disease, AHEI, SFA, fiber, sugar, fruit, vegetable, and energy intake. Multivariable linear regression models were used to calculate the effect sizes per I mg/g protein increment of tryptophan, adjusting for the same variables. Data are mean (SD).

Abbreviations: FPG, fasting plasma glucose; $\mathrm{HbA}_{\mathrm{Ic}}$, glycosylated hemoglobin; TC, total cholesterol; LDL-C, low-density lipoprotein cholesterol; APO-B, apolipoprotein B; BMI, body mass index; WHR, waist-to-hip ratio; AHEl, Alternate Healthy Eating Index; SFA, saturated fatty acid.

tryptophan had a reduced risk of developing MetSyn, compared with those in the lowest tertile. Furthermore, dietary tryptophan was positively associated with sleep duration, and negatively associated with FPG, $\mathrm{HbA}_{1 \mathrm{c}}$, TC, LDL-C, and APO-B. Sleep duration partially mediated the association between tryptophan and MetSyn. To the best of our knowledge, this is the first study to examine the effect of dietary tryptophan intake on MetSyn, and the mediating effect of sleep duration. Our study emphasizes the importance of a moderate increase in tryptophan consumption for MetSyn prevention and contributes toward a better understanding of the potential mechanisms by which the risks of MetSyn are mediated.

Our study found a negative association between tryptophan intake and MetSyn incidence in the CHNS. This association was robust even after adjusting for numerous potential confounders such as nutrients and food patterns.
Foods with a high tryptophan content include milk, eggs, meat, potatoes, and nuts. ${ }^{34}$ The cumulative amounts of meat, potatoes, eggs, milk, and nuts consumed in our study were $99.31,25.35,18.89,4.92$, and $2.34 \mathrm{~g} /$ day, respectively, which are parallel to other studies in Chinese populations. ${ }^{35-37}$ Because of the relatively low intake of milk and nuts in China, the main sources of tryptophan in our study may be meat, potatoes, and eggs. Research has demonstrated that tryptophan consumption may regulate the basic components of MetSyn, such as weight gain, glucose metabolism, blood pressure, and lipids, which substantiates our findings. ${ }^{38-41}$ Animal studies have revealed the potential mechanisms of these effects. Ardiansyah et al suggested that both a single oral administration and continuous treatment with tryptophan improved glucose metabolism and blood pressure in rats, which were mediated by serotonin levels. ${ }^{11}$ A similar

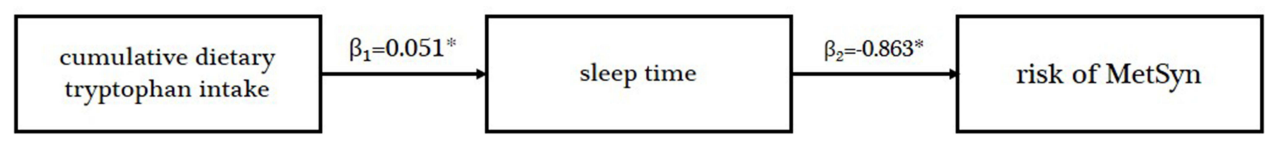

$\beta$ total effect $=-0.166^{*}$

Mediation effect by sleep time $=26.5 \% *$

Figure I Mediation effect of sleep time between cumulative dietary tryptophan intake and risk of MetSyn in the CHNS, I997-20II.

Notes: Data are standardized regression coefficients with adjustment for sex, age, BMI, WHR, smoking status, drinking status, living site, physical activity, urban index, cardiovascular disease, AHEI, SFA, fiber, sugar, fruit, vegetable, and energy intake. $* P<0.05$ for coefficients different from 0 .

Abbreviations: MetSyn, metabolic syndrome; BMI, body mass index; WHR, waist-to-hip ratio; AHEl, Alternate Healthy Eating Index; SFA, saturated fatty acid. 
influence was found on triglyceride levels. ${ }^{12}$ As for weight gain, Ayaso et al demonstrated that dietary tryptophan could reduce food intake and was then translated into a decrease in weight, which came from the effect of brain serotonin on satiety. ${ }^{42}$ On the other hand, the negative association between the central serotonergic system and MetSyn offers support to our study. ${ }^{43,44}$ Tryptophan is the substrate for serotonin synthesis and is converted to 5-hydroxytryptophan (5-HT) by tryptophan hydroxylase, which is the rate-limiting enzyme for the production of serotonin. ${ }^{45}$ Because tryptophan hydroxylase is $50 \%$ saturated with its substrate under normal physiological conditions, a change in tryptophan availability in the brain can significantly alter brain serotonin synthesis. ${ }^{46}$ The manipulation of dietary tryptophan influences plasma tryptophan levels and the tryptophan/large neutral amino acids (LNAA) ratio, and the tryptophan/LNAA ratio determines the availability of tryptophan across the blood-brain barrier. ${ }^{20}$ Serotonin-containing neurons are concentrated in the raphé nuclei of the brainstem and project diffusely to the cerebral cortex, hypothalamus, and major autonomic nuclei, where they appear to exert broad regulatory control. ${ }^{44}$ Disorders of the brain's serotonin signal may affect eating habits by accelerating the onset of satiety ${ }^{47}$ or energy expenditure via the stimulation of thermogenesis in brown adipose tissue, ${ }^{48}$ or both, leading indirectly to the development of obesity, insulin resistance, and related phenomena. ${ }^{49}$ Alternatively, central serotonin may act through the autonomic nervous system or hypothalamicpituitary axis to affect blood pressure and key metabolic processes. ${ }^{50,51}$ Therefore, it is rational that dietary tryptophan exerts an impact on the serotonin level and thus decreases the risk of MetSyn. The interaction between tryptophan and obesity/overweight in our study was likely explained by the fact that the up-regulation of indoleamine 2,3-dioxygenase (IDO) activity, essentially caused by chronic immune-mediated inflammation in obesity, resulted in decreased tryptophan availability for the serotonin pathway. ${ }^{52}$ On this basis, the protective effect on MetSyn was more prominent among participants who were not overweight.

The analysis of the association between tryptophan and sleep duration in our study indicated that tryptophan consumption could moderately increase sleep time under reallife conditions. Support for the advantageous impact of dietary tryptophan on sleep parameters comes from a meta-analysis including 18 studies from various ethnicities and regions, which demonstrated that tryptophan could improve multiple sleep parameters. ${ }^{19}$ However, the exact mechanism of the sedating effect of tryptophan has not been fully clarified. Tryptophan is the precursor for the neurotransmitter serotonin, which is an intermediary product in the synthesis of melatonin, both of which are believed to participate in the regulation of sleep and circadian rhythms, and are used to treat insomnia. ${ }^{53}$ Furthermore, previous studies in humans and experimental animals have convincingly documented the ability of dietary tryptophan to affect serotonin and melatonin secretion. ${ }^{54,55}$ Hence, tryptophan may exert its somnolent effect via the serotonin and melatonin pathway. ${ }^{56}$ One of the physiological effects of melatonin is stimulating peripheral vasodilation, which, in turn, decreases body core temperature. This lowering of the central temperature facilitates the onset of sleep. ${ }^{57}$ However, similarly to 5-HT, the exact mechanisms and area where serotonin and melatonin act to induce sleep are not known. Therefore, more research on the mechanisms is needed in the future.

Laboratory and epidemiological studies have demonstrated that short sleep duration is associated with, and may contribute toward, MetSyn. Spiegel et al presented several adverse effects of short sleep on glucose metabolism and energy homeostasis, which may be the potential mechanisms underlying the association between short sleep duration and MetSyn risk. The specific mechanism is still unknown, but the possible pathways linking short sleep to hypertension and cardiovascular disease events include weight gain and glucose metabolism disorder. ${ }^{13,18}$ These detrimental effects of short sleep duration involve multiple pathways. ${ }^{58,59}$ First, sympathetic and parasympathetic activity is elevated in short sleep duration, and this plays a key role in insulin and glucagon secretion, muscle insulin resistance, and adipocyte function. Second, cerebral glucose uptake by the "tired/sleepy" brain is decreased, which causes glucose metabolism disorder. Third, the hypothalamic-pituitary-adrenal (HPA) axis is activated, causing increases in growth hormone and cortisol levels, and then insulin resistance. Fourth, increased levels of inflammatory markers contribute toward insulin resistance. Finally, the dysregulation of neuroendocrine control of appetite leads to increased food intake and weight gain. Furthermore, moderately increasing the sleep time has a protective effect on MetSyn incidence. Compared to the shortest sleep duration, the odds ratio of the longest tertile of sleep duration was 0.80 (95\% CI: $0.68-0.94, P$ for trend=0.007) in our research (Supplementary Table 4). A study conducted by Duan et al also found that the adjusted ORs for MetSyn, abdominal 
obesity, high glucose, and high TG seemed to decrease with increment of sleep duration, and showed a linear doseresponse relationship between sleep duration and MetSyn and its components. ${ }^{60}$ Other research demonstrated unequivocally that extending sleep duration could increase leptin and TSH levels, decrease the sympathovagal balance and cortisol levels, and improve carbohydrate metabolism. ${ }^{13,61}$ The above research demonstrated the potential mechanism for the association of sleep duration with MetSyn risk, and provided the basis for the mediation effect of sleep.

Little attention has been paid to the relationship between dietary tryptophan and MetSyn risk in clinical practice, although MetSyn is closely linked to cardiovascular disease and diabetes. Our results showed the potential improvement with tryptophan intake on MetSyn incidence and related blood biomarkers, such as fasting glucose and TC, which may provide new clinical guidelines for MetSyn therapy. Strengths of our study include the prospective design, relatively large cohort size, and long follow-up duration. However, some limitations in the study need to be addressed. First, our study was conducted among Asian participants only, which is likely to limit the generalizability of our findings to other ethnic populations. Second, 24-hour recall was used to measure individual dietary intake in our study, which may not portray participants' typical intake precisely and could be subject to recall bias. However, the selection of field workers who are professionally engaged in nutrition work and the combination of consecutive 3-day 24-hour recall and the household food inventory could improve the accuracy of recall. Third, the detection of diseases was primarily based on self-report, with blood indices only being obtained in the 2009 survey, leading to incident cases being higher in the 2009 survey than in other surveys, and the incidence of MetSyn was relatively low throughout the CHNS, which may bias the Cox model results for time-event estimation. Fourth, the investigation of tryptophan supplementation was lacking in the CHNS, although this supplement was not common and was even rare. So, in our study we were not able to compare the doses of tryptophan obtained from the diet and from supplements. Future research is needed to explore the effects of tryptophan supplements. Furthermore, we focused on sleep duration in this study. Despite this being the most common sleep parameter, other dimensions of sleep should be considered in future studies.

\section{Conclusion}

Our study is the first one to propose the negative association between dietary tryptophan and the incidence of MetSyn, after adjusting variables such as nutrients and food patterns. Participants with the highest tertile of tryptophan consumption showed 23\% lower risk of MetSyn. Moreover, sleep duration partially mediated this association. The results of this large prospective study support a potential benefit of tryptophan intake in the prevention and management of MetSyn, which may be due to its effect in improving sleep. Because the underlying mechanisms of these phenomena remain elusive, further mechanistic studies exploring the impact of tryptophan consumption on MetSyn and its potential mediation by sleep are needed.

\section{Funding}

This work was supported by the National Natural Science Foundation (82030100 to Ying Li; 82073534 to Changhao Sun).

\section{Disclosure}

The authors report no conflicts of interest in this work.

\section{References}

1. Liu J, Grundy SM, Wang W, et al. Ethnic-specific criteria for the metabolic syndrome: evidence from China. Diabetes Care. 2006;29 (6):1414. doi:10.2337/dc06-0481

2. Panel on Detection, Evaluation, and Treatment of High Blood Cholesterol in Adults. Executive summary of the third report of the national cholesterol education program (NCEP) expert panel on detection, evaluation, and treatment of high blood cholesterol in adults (adult treatment panel III). JAMA. 2001;285(19):2486-2497. doi:10.1001/jama.285.19.2486

3. Hirode G, Wong RJ. Trends in the prevalence of metabolic syndrome in the United States, 2011-2016. JAMA. 2020;323(24):2526-2528. doi:10.1001/jama.2020.4501

4. Lutsey PL, Steffen LM, Stevens J. Dietary intake and the development of the metabolic syndrome. Circulation. 2008;117(6):754-761. doi:10.1161/CIRCULATIONAHA.107.716159

5. Heshmati J, Sepidarkish M, Namazi N, et al. Impact of dietary calcium supplement on circulating lipoprotein concentrations and atherogenic indices in overweight and obese individuals: a systematic review. J Diet Suppl. 2019;16(3):357-367. doi:10.1080/ 19390211.2018.1440685

6. Pucci G, Alcidi R, Tap L, Battista F, Mattace-Raso F, Schillaci G. Sexand gender-related prevalence, cardiovascular risk and therapeutic approach in metabolic syndrome: a review of the literature. Pharmacol Res. 2017;120:34-42. doi:10.1016/j.phrs.2017.03.008

7. Fernstrom JD, Wurtman RJ. Brain serotonin content: physiological dependence on plasma tryptophan levels. Science. 1971;173 (3992):149. doi:10.1126/science.173.3992.149

8. Lam DD, Heisler LK. Serotonin and energy balance: molecular mechanisms and implications for type 2 diabetes. Expert Rev Mol Med. 2007;9(5):1-24. doi:10.1017/S1462399407000245 
9. Jacobs BL, Azmitia EC. Structure and function of the brain serotonin system. Physiol Rev. 1992;72(1):165-229. doi:10.1152/physrev.1992. 72.1 .165

10. Lucki I. The spectrum of behaviors influenced by serotonin. Biol Psychiatry. 1998;44(3):151-162. doi:10.1016/S0006-3223(98) 00139-5

11. Ardiansyah S, Shirakawa H, Inagawa Y, Koseki T, Komai M. Regulation of blood pressure and glucose metabolism induced by L- tryptophan in stroke-prone spontaneously hypertensive rats. Nutr Metab. 2011;8(1):45. doi:10.1186/1743-7075-8-45

12. Fears R, Murrellt EA. Tryptophan and the control of triglyceride and carbohydrate metabolism in the rat. Br J Nutr. 1980;43(2):349-356. doi:10.1079/BJN19800097

13. Spiegel K, Tasali E, Leproult R, Van Cauter E. Effects of poor and short sleep on glucose metabolism and obesity risk. Nat Rev Endocrinol. 2009;5(5):253-261. doi:10.1038/nrendo.2009.23

14. Chung K-F, Yeung W-F, Ho F-Y-Y, Yung K-P, Yu Y-M, Kwok C-W. Cross-cultural and comparative epidemiology of insomnia: the diagnostic and statistical manual (DSM), international classification of diseases (ICD) and international classification of sleep disorders (ICSD). Sleep Med. 2015;16(4):477-482. doi:10.1016/j. sleep.2014.10.018

15. Cao XL, Wang SB, Zhong BL, et al. The prevalence of insomnia in the general population in China: a meta-analysis. PLoS One. 2017;12 (2):e0170772. doi:10.1371/journal.pone.0170772

16. Calhoun DA, Harding SM. Sleep and hypertension. Chest. 2010;138 (2):434-443. doi:10.1378/chest.09-2954

17. Kopelman PG. Obesity as a medical problem. Nature. 2000;404 (6778):635-643. doi:10.1038/35007508

18. Xi B, He D, Zhang M, Xue J, Zhou D. Short sleep duration predicts risk of metabolic syndrome: a systematic review and meta-analysis. Sleep Med Rev. 2014;18(4):293-297. doi:10.1016/j.smrv.2013.06.001

19. Sutanto CN, Loh WW, Kim JE. The impact of tryptophan supplementation on sleep quality: a systematic review, meta-analysis, and meta-regression. Nutr Rev. 2021. doi:10.1093/nutrit/nuab027

20. Silber BY, Schmitt JAJ. Effects of tryptophan loading on human cognition, mood, and sleep. Neurosci Biobehav Rev. 2010;34 (3):387-407. doi:10.1016/j.neubiorev.2009.08.005

21. Lucht S, Hennig F, Moebus S, et al. All-source and source-specific air pollution and 10-year diabetes incidence: total effect and mediation analyses in the Heinz Nixdorf recall study. Environ Int. 2020;136:105493.

22. Popkin BM, Du S, Zhai F, Zhang B. Cohort profile: the China health and nutrition survey-monitoring and understanding socio-economic and health change in China, 1989-2011. Int J Epidemiol. 2010;39 (6):1435-1440. doi:10.1093/ije/dyp322

23. Chen CM. Nutrition status of the Chinese people. Biomed Environ Sci. 1996;9(2-3):81-92.

24. Li YP, He YN, Zhai FY, et al. Comparison of assessment of food intakes by using 3 dietary survey methods. Zhonghua Yu Fang Yi Xue Za Zhi. 2006;40(4):273-280.

25. Zhai FY, Du SF, Wang ZH, Zhang JG, Du WW, Popkin BM. Dynamics of the Chinese diet and the role of urbanicity, 19912011. Obes Rev. 2014;15(S1):16-26. doi:10.1111/obr.12124

26. Foster E, Lee C, Imamura F, et al. Validity and reliability of an online self-report 24-h dietary recall method (Intake24): a doubly labelled water study and repeated-measures analysis. J Nutr Sci. 2019;8:e29. doi:10.1017/jns.2019.20

27. Xu X, Byles JE, Shi Z, Hall JJ. Evaluation of older Chinese people's macronutrient intake status: results from the China Health and Nutrition Survey. Br J Nutr. 2015;113(1):159-171. doi:10.1017/ S0007114514003444

28. Ng SW, Howard AG, Wang HJ, Su C, Zhang B. The physical activity transition among adults in China: 1991-2011. Obes Rev. 2014;15 (S1):27-36. doi:10.1111/obr.12127
29. Lu J, Wang L, Li M, et al. Metabolic syndrome among adults in China: the 2010 China noncommunicable disease surveillance. J Clin Endocrinol Metab. 2017;102(2):507-515.

30. Grundy SM, Cleeman JI, Daniels SR, et al. Diagnosis and management of the metabolic syndrome. Circulation. 2005;112 (17):2735-2752. doi:10.1161/CIRCULATIONAHA.105.169404

31. Qin LQ, Xun P, Bujnowski D, et al. Higher branched-chain amino acid intake is associated with a lower prevalence of being overweight or obese in middle-aged East Asian and Western adults. J Nutr. 2011;141(2):249-254. doi:10.3945/jn.110.128520

32. Ananthakrishnan AN, Khalili H, Konijeti GG, et al. A prospective study of long-term intake of dietary fiber and risk of crohn's disease and ulcerative colitis. Gastroenterology. 2013;145(5):970-977. doi:10.1053/j.gastro.2013.07.050

33. Rosseel Y. lavaan: an $\mathrm{R}$ package for structural equation modeling. J Stat Softw. 2012;48(2):36.

34. Palego L, Betti L, Rossi A, Giannaccini GJ. Tryptophan biochemistry: structural, nutritional, metabolic, and medical aspects in humans. J Amino Acids. 2016;2016. doi:10.1155/2016/8952520

35. Liu AD, Zhang B, Wang HJ, Su C, Zhai FY. Milk consumption and its changing trend of Chinese adult aged 18-44 in nine provinces (autonomous region) from 1991 to 2006. Zhonghua Yu Fang Yi Xue Za Zhi [Chinese Journal of Preventive Medicine]. 2011;45 (4):304-309.

36. Bing Chen B, Han Y, Pan X, et al. Association between nut intake and non-alcoholic fatty liver disease risk: a retrospective case-control study in a sample of Chinese Han adults. BMJ Open. 2019;9(9): e028961. doi:10.1136/bmjopen-2019-028961

37. Xin L-J, Li P-H. Food consumption patterns of Chinese urban and rural residents based on CHNS and comparison with the data of National Bureau of Statistics. J Nat Sci. 2018;33(1):75-84.

38. Cade JR, Fregly MJ, Privette M. Effect of tryptophan and 5-hydroxytryptophan on the blood pressure of patients with mild to moderate hypertension. Amino Acids. 1992;2:133-142. doi:10.1007/ BF00806084

39. Cade JR, Fregly MJ, Privette M. Effect of L-tryptophan on the blood pressure of patients with mild to moderate essential hypertension. In: Lubec G, Rosenthal GA, editors. Amino Acids: Chemistry, Biology and Medicine. Dordrecht: Springer Netherlands; 1990:738-744.

40. Hajishafiee M, Ullrich SS, Steinert RE, et al. Effects of intragastric tryptophan on acute changes in the plasma tryptophan/large neutral amino acids ratio and relationship with subsequent energy intake in lean and obese men. Food Funct. 2020;11(8):7095-7103. doi:10.1039/D0FO00773K

41. Steinert RE, Luscombe-Marsh ND, Little TJ, et al. Effects of intraduodenal infusion of L-tryptophan on ad libitum eating, antropyloroduodenal motility, glycemia, insulinemia, and gut peptide secretion in healthy men. J Clin Endocrinol Metab. 2014;99(9):3275-3284. doi:10.1210/jc.2014-1943

42. Ayaso R, Ghattas H, Abiad M, Obeid O. Meal pattern of male rats maintained on amino acid supplemented diets: the effect of tryptophan, lysine, arginine, proline and threonine. Nutrients. 2014;6:7. doi:10.3390/nu6072509

43. Muldoon MF, Mackey RH, Korytkowski MT, Flory JD, Pollock BG, Manuck SB. The metabolic syndrome is associated with reduced central serotonergic responsivity in healthy community volunteers. J Clin Endocrinol Metab. 2006;91(2):718-721. doi:10.1210/jc.2005-1654

44. Muldoon MF, Mackey RH, Williams KV, Korytkowski MT, Flory JD, Manuck SB. Low central nervous system serotonergic responsivity is associated with the metabolic syndrome and physical inactivity. $J$ Clin Endocrinol Metab. 2004;89(1):266-271. doi:10.1210/jc.2003-031295

45. Fernstrom JD. A perspective on the safety of supplemental tryptophan based on its metabolic fates. J Nutr. 2016;146(12):2601S2608S. doi:10.3945/jn. 115.228643 
46. Fernstrom JD. Role of precursor availability in control of monoamine biosynthesis in brain. Physiol Rev. 1983;63(2):484-546. doi:10.1152/ physrev.1983.63.2.484

47. Kałużna-Czaplińska J, Gątarek P, Chirumbolo S, Chartrand MS, Bjørklund G. How important is tryptophan in human health? Crit Rev Food Sci Nutr. 2019;59(1):72-88. doi:10.1080/10408398.2017.1357534

48. McGlashon Jacob M, Gorecki Michelle C, Kozlowski Amanda E, et al. Central serotonergic neurons activate and recruit thermogenic brown and beige fat and regulate glucose and lipid homeostasis. Cell Metab. 2015;21(5):692-705. doi:10.1016/j.cmet.2015.04.008

49. Donovan M, Tecott L. Serotonin and the regulation of mammalian energy balance. Front Neurosci. 2013;7:36. doi:10.3389/ fnins.2013.00036

50. McCall RB, Clement ME. Role of serotonin1A and serotonin2 receptors in the central regulation of the cardiovascular system. Pharmacol Rev. 1994;46(3):231.

51. Fuller RW. Serotonin receptors and neuroendocrine responses. Neuropsychopharmacology. 1990;3(5-6):495-502.

52. Mangge H, Summers KL, Meinitzer A, et al. Obesity-related dysregulation of the Tryptophan-Kynurenine metabolism: role of age and parameters of the metabolic syndrome. Obesity. 2014;22(1):195-201. doi:10.1002/oby.20491

53. Lieberman HR, Agarwal S, Fulgoni VL. Tryptophan intake in the US adult population is not related to liver or kidney function but is associated with depression and sleep outcomes. J Nutr. 2016;146 (12):2609S-2615S. doi:10.3945/jn.115.226969

54. Esteban S, Nicolaus C, Garmundi A, et al. Effect of orally administered 1-tryptophan on serotonin, melatonin, and the innate immune response in the rat. Mol Cell Biochem. 2004;267(1):39-46. doi:10.1023/B:MCBI.0000049363.97713.74
55. Zimmermann RC, McDougle CJ, Schumacher M, et al. Effects of acute tryptophan depletion on nocturnal melatonin secretion in humans. J Clin Endocrinol Metab. 1993;76(5):1160-1164.

56. Levitan RD, Shen JH, Jindal R, Driver HS, Kennedy SH, Shapiro CM. Preliminary randomized double-blind placebo-controlled trial of tryptophan combined with fluoxetine to treat major depressive disorder: antidepressant and hypnotic effects. J Psychiatry Neurosci. 2000;25 (4):337-346.

57. Dawson D, Encel N. Melatonin and sleep in humans. J Pineal Res. 1993;15(1):1-12. doi:10.1111/j.1600-079X.1993.tb00503.x

58. Tasali E, Leproult R, Spiegel K. Reduced sleep duration or quality: relationships with insulin resistance and type 2 diabetes. Prog Cardiovasc Dis. 2009;51(5):381-391. doi:10.1016/j.pcad.2008. 10.002

59. Reutrakul S, Van Cauter E. Sleep influences on obesity, insulin resistance, and risk of type 2 diabetes. Metabolism. 2018;84:56-66. doi:10.1016/j.metabol.2018.02.010

60. Duan Y, Sun J, Wang M, Zhao M, Magnussen CG, Xi B. Association between short sleep duration and metabolic syndrome in Chinese children and adolescents. Sleep Med. 2020;74:343-348. doi:10.1016/j.sleep.2020.08.018

61. Spiegel K, Leproult R, L'Hermite-Balériaux M, Copinschi G, Penev PD, Van Cauter E. Leptin levels are dependent on sleep duration: relationships with sympathovagal balance, carbohydrate regulation, cortisol, and thyrotropin. J Clin Endocrinol Metab. 2004;89(11):5762-5771. doi:10.1210/jc.2004-1003

\section{Publish your work in this journal}

Nature and Science of Sleep is an international, peer-reviewed, open access journal covering all aspects of sleep science and sleep medicine, including the neurophysiology and functions of sleep, the genetics of sleep, sleep and society, biological rhythms, dreaming, sleep disorders and therapy, and strategies to optimize healthy sleep.
The manuscript management system is completely online and includes a very quick and fair peer-review system, which is all easy to use. Visit http://www.dovepress.com/testimonials.php to read real quotes from published authors. 